\title{
Human Colostrum Has Anti-inflammatory Activity in a Rat Subcutaneous Air Pouch Model of Inflammation
}

\author{
DONALD K. MURPHEY AND E. STEPHEN BUESCHER \\ University of Texas Medical School at Houston. Department of Pediatrics. Division of Infectious Diseases, \\ Houston. Texas 78705 (D.K.M.J; and Center for Pediatric Research. Eastern Virginia Medical School, \\ Norfolk, Virginia 23510 /E.S.B./
}

\begin{abstract}
An animal model was used to examine the effect of human colostrum on an acute inflammatory process in vivo. Subcutaneous air pouches on the backs of outbred rats were injected with carrageenan as an inflammatory challenge, normal saline, pooled aqueous human colostrum, or carrageenan plus colostrum concurrently. Oral dexamethasone or indomethacin was administered to some animals before and during challenge as anti-inflammatory agents. Polymorphonuclear leukocyte (PMN) counts in pouch fluid were determined $6 \mathrm{~h}$ postchallenge. Carrageenan challenge resulted in a significant acute inflammatory response $\left[48.8 \pm 4.9 \times 10^{6} \mathrm{PMN} /\right.$ pouch (mean \pm SEM, $n=46) \mid$ compared with normal saline controls $\left[0.9 \pm 0.2 \times 10^{6}(n=31, p<0.001\right.$ versus carrageenan $\left.)\right]$ or with colostrum $\left[4.3 \pm 0.8 \times 10^{6} \mathrm{PMN} /\right.$ pouch $(n=11$, $p<0.001$ versus carrageenan)]. The concurrent injection of colostrum plus carrageenan challenge significantly reduced the PMN response compared with carrageenan alone $\left[18.8 \pm 2.9\right.$ versus $48.8 \pm 4.9 \times 10^{6} \mathrm{PMN} /$ pouch (carrageenan plus colostrum versus carrageenan, $n=41$ versus 46, $p<0.001)$ ]. This degree of suppression of PMN influx was not significantly different from that seen with indomethacin treatment but was significantly more than that seen with dexamethasone treatment. The decreases in PMN counts observed most likely reflect suppression of the acute inflammatory response because a significant amount of PMN lysis in colostrum was not observed in vitro and the accumulation of PMN granule contents was not seen in pouch fluid from colostrum-treated animals in vivo. These data directly demonstrate for the first time that human colostrum has a biologically significant effect on the inflammatory process in vivo. (Pediatr Res 34: 208212,1993 )
\end{abstract}

\section{Abbreviations}

Carr, $0.2 \%$ solution of carrageenan in PBS

Col, pooled aqueous human colostrum

PMN, polymorphonuclear leukocyte

Carr + Col, concurrent challenge with Carr and Col

HBSS, Hanks' balanced salt solution without calcium or magnesium

Human colostrum contains an average of $1.5 \times 10^{9} \mathrm{PMN} / \mathrm{L}$ over the first $4 \mathrm{~d}$ of lactation and nearly twice this number of

Received November 13. 1992: accepted March 11. 1993

Correspondence and reprint requests: Donald K. Murphey, M.D.. Infectious Diseases, Cook-Fort Worth Children's Medical Center, 801 Seventh Avenue, Fort Worth, TX 76104 .

Supported by National Institute of Child Health and Human Development Grant HD 13021-09. total leukocytes $(1,2)$. Human newborns, assuming an average oral intake of about $0.1 \mathrm{~L} / \mathrm{kg} / \mathrm{d}$ over this period, will consume more than 2 billion PMN and as many mononuclear cells. These cells may offer the newborn some benefit by augmenting its immature host defenses. However, leukocytes in colostrum and breast milk function poorly compared with circulating white blood cells (3-9), making it difficult to understand how they could augment host defenses. However, if the primary role of these maternal cells were to protect a mother's breasts as this new secretory pathway was established, then these cells may offer little immunologic benefit to the infant directly. If the PMN in colostrum continued to function normally, they might pose a threat to the infant's gastrointestinal tract mucosa because they could mediate inflammation and local tissue injury (10-13).

We hypothesize that antileukocyte activity in colostrum is needed to protect the newborn in this unique physiologic setting of mucosal exposure to exogenous PMN and that this antileukocyte activity results in a biologically significant anti-inflammatory effect. In vitro data directly demonstrate that human colostrum and milk have antileukocyte activity. Human milk and colostrum inhibit many peripheral blood $(3-5,14-16)$ and milk-derived leukocyte (3-9) functions in vitro. Human colostrum can protect cell monolayers from injury by activated PMN, and this protection is probably mediated by several antioxidant components $(17,18)$.

Inhibition of PMN activity by colostrum might be expected to result in suppression of acute inflammatory responses. Acute inflammation is a dynamic process involving tissue and circulating leukocytes, and the best evidence of a biologically significant anti-inflammatory effect is based on data from epidemiologic or in vivo experimental data. An anti-inflammatory effect of human colostrum or breast milk in vivo has not been described; however, in humans, several epidemiologic phenomena suggest such an effect. Common respiratory and gastrointestinal infections are less frequent and severe in infants who are breast-fed $(19,20)$. This protection may be mediated by specific anti-infective factors in milk, such as secretory immunoglobulin, lysozyme, lactoferrin, and oligosaccharides $(21,22)$. However, these infections represent the most common stimulus for inflammation in this population, and an anti-inflammatory effect of breast-feeding might be indistinguishable from an anti-infective effect. Enteric infection with pathogens that typically produce intestinal inflammation, such as Shigella, are commonly noninflammatory in breast-fed infants $(23,24)$. Necrotizing enterocolitis also has been shown to be significantly less likely to occur in breast-fed infants (25). Although indirect, these associations suggest the possibility of anti-inflammatory activity in breast milk. Reviewing the available data, other investigators have found support for the hypothesis that breast milk is anti-inflammatory $(19,20)$.

A well-described animal model of acute inflammation is used to examine whether human colostrum can alter the cascade of 
events in acute inflammation leading to PMN accumulation locally and to compare the magnitude of this effect with those of known potent anti-inflammatory agents.

\section{MATERIALS AND METHODS}

Collection of colostrum. Colostrum used for these studies was the lactational product obtained from inpatient mothers from 1 to $4 \mathrm{~d}$ postpartum after informed consent was obtained. No mothers had symptoms of mastitis. Mothers were not excluded because of premature delivery. The entire contents of one breast were obtained aseptically using an electric breast pump (Egnell, Cary, IL). Colostrum was immediately chilled and transported to the laboratory on ice. Specimens were then promptly separated by ultracentrifugation (LS-70, Beckman Instruments, Inc.. Palo Alto, CA) at $81000 \times g$ for $30 \mathrm{~min}$ at $4^{\circ} \mathrm{C}$. The aqueous fraction was collected by puncturing the centrifuge tube with an 18-gauge needle and draining this fraction dropwise into a sterile plastic container. The cream layer and cell pellet were discarded, and the samples were frozen at $-70^{\circ} \mathrm{C}$ until used. Samples of aqueous colostrum from at least six donors were thawed and pooled. Each pool of colostrum was filter-sterilized $(0.45 \mu \mathrm{m})$ and stored at $-70^{\circ} \mathrm{C}$ until used. Seven pools were used for these experiments. Individual donor colostrum was prepared similarly but not pooled.

Challenge materials. All challenge materials were prepared aseptically. PBS was prepared with $10 \times$ PBS (Whittaker, M. A. Bioproducts. Walkersville, MD) and charcoal-filtered, nonsterile water, then autoclaved. Normal saline for injection (Baxter Healthcare Corp., Deerfield. IL) was aspirated from a sealed bag immediately before use. Carrageenan (type IV, Sigma Chemical Co., St. Louis, MO) was dissolved in PBS at $2 \mathrm{~g} / \mathrm{L}$ using vigorous agitation and autoclaving and was stored at $4^{\circ} \mathrm{C}$. Carrageenan solutions (Carr) were agitated and warmed to $20^{\circ} \mathrm{C}$ before use. Carrageenan solutions with visible precipitate were reautoclaved and cooled before use.

Rat model of aculc inflammation. The protocol described was approved by the Animal Welfare Committee of the University of Texas Health Science Center Houston. Female outbred Sprague-Dawley rats (Sasco. Omaha, NE), weighing 125-150 g. were housed six to a cage in a temperature- and ventilationcontrolled environment and given standard rat food and water ad libitum. On d 0 after inducing $\mathrm{CO}_{2}$ necrosis, a $20-\mathrm{mL}$ subcutaneous air pouch was formed in the midline of the back of each rat by injection of air, and pouches were refilled with $10 \mathrm{~mL}$ of air in a similar manner. Tails were marked for identification on d 3. On d $6,5 \mathrm{~mL}$ of a challenge material, carrageenan, normal saline, or colostrum, were injected into the pouch. The effect of colostrum on inflammation was tested by injecting $5 \mathrm{~mL}$ of colostrum into the pouch immediately after carrageenan injection (Carr $+\mathrm{Col})$. Animals were observed periodically for signs of local or systemic distress. Six h later, rats were killed using $\mathrm{CO}_{2}$ overdose and pouch fluid was promptly collected. Pouches were injected with $3 \mathrm{~mL}$ of heparinized PBS $\left(10^{6} \mathrm{U} / \mathrm{L}\right)$ and vigorously kneaded for $15 \mathrm{~s}$. Skin overlying the pouch was shaved with electric clippers (Oster, no. 40 blade, Milwaukee, WI). and the pouch was opened with sharp dissection. Pouch fluid was drained and expressed into a plastic cup. The volume of fluid obtained was measured, and samples of pouch fluid were aliquoted. One aliquot of pouch fluid was diluted 1:10 with $10 \%$ formalin in PBS, and cell number determined using a hemocytometer. Differential cell counts were performed using cytocentrifuge-prepared slides (Shandon. Cheshire, England) of a fresh aliquot of pouch fluid, stained with a modified Wright's stain (Diff-Quik, Baxter, Miami, FL). In some cases, an aliquot of fluid was frozen at $-70^{\circ} \mathrm{C}$ for assay of PMN granule contents at a later time. Scrapings and histologic examination of the pouch wall after pouch fluid collection showed no adherent white blood cells (wbc) on the pouch wall surface. The number of PMN per pouch was calculated as follows:

PMN/pouch $\left(\times 10^{6}\right)=$ Volume $(\mathrm{mL})$ $\times$ cell number $\left(\times 10^{6} / \mathrm{mL}\right) \times$ wbc/cells $\times \mathrm{PMN} / \mathrm{wbc}$

Anti-inflammatory agents. Dexamethasone (Decadron elixir) and indomethacin (Indocin oral suspension) were gifts of Merck Sharp \& Dohme (West Point. PA). Either agent, dexamethasone $(10 \mathrm{mg} / \mathrm{L})$ or indomethacin $(100 \mathrm{mg} / \mathrm{L})$, was added to the animals' water supply for $3 \mathrm{~d}$ before and during challenge with carrageenan.

Cell stability in colostrum. Human PMN were purified from heparinized whole blood of healthy adult volunteers by centrifugation over a Ficoll-Hypaque gradient, dextran sedimentation, and hypotonic lysis (26) and resuspended at $10^{11}$ cells/L in HBSS. Autologous plasma diluted with one volume of HBSS was collected from the tops of the gradients after centrifugation and was filter-sterilized $(0.45 \mu \mathrm{m})$. Thawed pooled aqueous colostrum was similarly diluted and filtered. Triplicate samples prepared with $0.2 \mathrm{~mL}$ of PMN suspension and $3 \mathrm{~mL}$ of colostrum or autologous plasma were incubated in $5 \%$ moist $\mathrm{CO}_{2}$ at $37^{\circ} \mathrm{C}$. Aliquots were taken at various time points, and absolute and differential cell counts were performed as for pouch fluid.

Leukocyte granule marker assays. Aliquots of frozen pouch fluid were thawed and sonicated (W185. Heat Systems-Ultrasonic. Plainview, NY) at $40 \mathrm{~W}$ for $30 \mathrm{~s}$ on ice after the addition of equal volumes HBSS- $0.1 \%$ Triton X-100. Lysate supernatants were collected after centrifugation at $15000 \times g$ for $20 \mathrm{~min}$ at $4^{\circ} \mathrm{C}$ and stored at $-70^{\circ} \mathrm{C}$ until used. $\beta$-Glucuronidase activity was determined as previously described (27) by incubating 0.05 $\mathrm{mL}$ of lysate supernatant with $0.05 \mathrm{~mL} 2 \mathrm{mM}$ 4-methylumbelliferyl- $\beta$-D-glucuronide (Sigma) in $100 \mathrm{mM}$ acetate buffer $(\mathrm{pH}$ $3.5)-0.1 \%$ Triton $X-100$ at $37^{\circ} \mathrm{C}$ for $60 \mathrm{~min}$. and then stopping the reaction with $2.4 \mathrm{~mL}$ of $50 \mathrm{mM}$ glycine buffer ( $\mathrm{pH} \mathrm{10.4)-5}$ $\mathrm{mM}$ EDTA. Fluorescence of the samples was measured in a fluorescence spectrophotometer (650-10S, Perkin-Elmer, Oak Brook, IL), using 365-nm excitation and $450-\mathrm{nm}$ emission wavelengths. The correlation between fluorescence and PMN number was excellent ( $r=0.997)$ over a range of 0.1 to $35 \times 10^{9}$ purified human PMN/L in HBSS.

Fractionation of colostrum. A pool of aqueous colostrum was thawed and diluted into two portions: one portion was fractionated whereas the other was not. The fractionated portion was separated by molecular size filtration into three fractions containing components with $>100000-\mathrm{D}, 100000-$ to $10000-\mathrm{D}$. and $<10000-\mathrm{D}$ molecular mass ranges. This portion was partially passed through a filter retaining molecules $>100000 \mathrm{D}$ (YM100, Diaflow ultrafilters. Amicon, Beverly. MA) under nitrogen at $4^{\circ} \mathrm{C}$ with constant stirring, and the filtrate was collected. The retentate was washed once with one volume of normal saline when its volume reached $10 \%$ of original, and then this retentate was rediluted to the original starting volume with normal saline to produce the $>100000-\mathrm{D}$ fraction. The YM100 filtrate was refiltered and washed using a 10000 -D cutoff filter (YMI0), and the filtrate collected. The $100000-$ to $10000-\mathrm{D}$ fraction was prepared from the washed YM10 retentate in the manner described previously. The YM 10 filtrate was lyophilized (Cryolyzer, New Brunswick Scientific Co.. Inc., New Brunswick. NJ) and reconstituted with normal saline to the original sample volume to produce the $<10000-\mathrm{D}$ fraction. The unfractionated portion of colostrum and the prepared fractions were stored together during this procedure and were frozen together at $-70^{\circ} \mathrm{C}$ on completion.

Data analysis. Unless otherwise noted, data are expressed as means \pm SEM, and differences among groups were compared using $l$ test, with $p<0.05$ taken as a significant difference. Values of $n$ represent the numbers of animals used to examine each experimental condition. Linear regression analysis was used to compare PMN granule marker activity and PMN counts. 


\section{RESULTS}

Response to challenge materials in pouch fluid. Combined data from all experiments showing pouch fluid characteristics for animals challenged with normal saline, colostrum, carrageenan, and carrageenan plus colostrum are shown in Table 1. Animals with pouches injected with normal saline had no visible change in behavior, no signs of local inflammation in the skin overlying the pouch, and little PMN influx into pouch fluid. Pouch fluid cell counts were $0.9 \pm 0.2 \times 10^{6} \mathrm{PMN} /$ pouch for normal saline $(n=31)$ over many experiments. Injection of colostrum resulted in no changes in rat behavior, no signs of local inflammation, and a modest PMN influx. Pouch fluid PMN counts were $4.3 \pm$ $0.8 \times 10^{6} \mathrm{PMN} /$ pouch for colostrum $(n=11)(p<0.001$ for Col versus Carr or normal saline). In a single pilot trial, the use of whole or acellular colostrum resulted in more PMN influx (10.1 or $9.8 \times 10^{6} \mathrm{PMN} /$ pouch, respectively) than aqueous colostrum $\left(4.5 \times 10^{6} \mathrm{PMN} /\right.$ pouch $)$. Injection of either of these cream-containing materials into the pouch resulted in the accumulation of many lipid-laden white blood cells, which made accurate cell counting difficult. Additional experiments were performed only with Col.

Approximately half the animals challenged with carrageenan showed decreased activity or ruffled fur but very few showed warmth or tenderness over the pouch. These animals consistently demonstrated the accumulation of large numbers of PMN in pouch fluid. Pouch fluid PMN counts $6 \mathrm{~h}$ after carrageenan challenge were $48.4 \pm 4.9 \times 10^{6} \mathrm{PMN} /$ pouch $(n=46, p<0.001$ versus normal saline or $\mathrm{Col})$. This significant cellular influx consisted almost exclusively of PMN $(81 \pm 2 \%)$. No significant differences in PMN responses were seen when comparing carrageenan prepared in PBS or normal saline (data not shown).

Effects of aqueous colostrum on acute inflammation. Injection of colostrum immediately after carrageenan did not perceptibly alter the occurrence of behavioral changes compared with carrageenan-challenged animals. The addition of colostrum to the pouch consistently and significantly suppressed the influx of PMN into pouch fluid in response to carrageenan. Pouch fluid PMN counts were reduced by an average of $61 \%$ over the course of many experiments [18.8 \pm 2.9 versus $48.8 \pm 4.9 \times 10^{6} \mathrm{PMN} /$ pouch (Carr + Col, $n=41$, versus Carr, $n=46 ; p<0.001)]$.

Relative activities of colostrum and anti-inflammatory agents. Treatment with an anti-inflammatory agent before and during challenge with carrageenan significantly suppressed PMN influx into the pouch. Pouch fluid characteristics for animals concurrently challenged with carrageenan with or without anti-inflammatory agents or colostrum are shown in Table 2. Pouch fluid PMN counts for dexamethasone-treated animals were reduced by an average of $64 \%\left[18.9 \pm 3.3\right.$ versus $53.2 \pm 6.6 \times 10^{6} \mathrm{PMN} /$ pouch (Carr + dexamethasone versus Carr, $n=16$ each, $p<$ 0.001 versus Carr)]. Pouch fluid PMN counts for indomethacintreated animals were reduced by an average of $79 \%, 8.7 \pm 3.0 \times$ $10^{6} \mathrm{PMN} /$ pouch (Carr + indomethacin, $n=13, p<0.001$ versus Carr). Pouch fluid PMN counts for colostrum-treated animals were reduced by an average of $85 \%, 8.0 \pm 2.9 \times 10^{6} \mathrm{PMN} /$ pouch (Carr $+\mathrm{Col}, n=13, p<0.001$ versus Carr). Treatment with colostrum or indomethacin was significantly more effective in reducing PMN influx into pouch fluid after carrageenan challenge than dexamethasone $(p<0.05)$.

Variability in carrageenan activity. Six carrageenan preparations were used during the course of these experiments, and variability in the inflammatory activity of these preparations was seen. Pouch fluid PMN counts ranged from $82.7 \pm 9.7 \times 10^{6}$ $\mathrm{PMN} /$ pouch $(n=9)$ for the most active preparation to $13.2 \pm$ $8.2 \times 10^{6} \mathrm{PMN} /$ pouch $(n=4)$ for the least active preparation. This variation may be related to the poor-solubility of carrageenan in water. Precipitation was seen in the preparation with the least activity, and this precipitate cleared and activity improved after reautoclaving the preparation.

Variability of colostral anti-inflammatory activity. A comparison of PMN responses in paired carrageenan and carrageenan plus colostrum-treated animals was made for each carrageenan preparation used. Colostrum treatment resulted in mean decreases in pouch fluid PMN counts of 22 to $85 \%$ in each carrageenan preparation examined, and these differences were significant for three of the six preparations. Three of four preparations with seven or more animals in each carrageenan and colostrum plus carrageenan group produced significant differences, demonstrating that larger groups tended to show significant differences, as expected. Three of four carrageenan preparations with $\mathrm{PMN}$ responses of less than $50 \times 10^{6} \mathrm{PMN} /$ pouch failed to show significant differences between carrageenan and carrageenan plus colostrum-treated animals, demonstrating that a less inflammatory carrageenan preparation more often failed to demonstrate significant suppression.

Seven pools of aqueous colostrum were used during the course of these experiments. PMN responses among paired carrageenan and carrageenan plus colostrum-treated animals were compared for each colostrum pool used. Six of seven colostrum pools produced mean decreases in pouch fluid PMN counts of 50 to $84 \%$, whereas one pool showed a $10 \%$ decrease. Significant suppression of PMN influx was present in four of seven pools.

In a single experiment, aqueous colostrum samples from four individual donors were compared with a colostrum pool prepared from other donors. The pooled colostrum reduced the PMN influx in response to carrageenan by $52 \%$, whereas the individual donor samples reduced the influx by $26,27,33$, and $74 \%$. These data indicate that anti-inflammatory activity in colostrum may vary considerably among single samples from single donors. The consistent anti-inflammatory activity in several pools of colostrum suggests that pooling six or more individual donor's colostrum samples minimizes the variability among single donor samples.

PMN stability in colostrum. The possibility that lysis of PMN in the presence of colostrum was responsible for the decreased PMN counts observed was evaluated. When human PMN were incubated in either pooled aqueous colostrum or autologous plasma, cell counts decreased from 6.45 to $6.35 \times 10^{9} \mathrm{PMN} / \mathrm{L}$ in plasma (average of triplicate determinations) and from 7.83 to $6.90 \times 10^{9} \mathrm{PMN} / \mathrm{L}$ in colostrum over $6 \mathrm{~h}$ of incubation. Cell counts at $24 \mathrm{~h}$ of incubation were $3.18 \times 10^{9} \mathrm{PMN} / \mathrm{L}$ in plasma

Table 1. Acute inflammatory responses to challenge materials and anti-inflammatory effect of Col in rat air pouch model of inflammation*

\begin{tabular}{lcccc}
\hline \multicolumn{1}{c}{ Pouch fluid } & $\begin{array}{c}\text { Normal saline } \\
(n=31)\end{array}$ & $\begin{array}{c}\text { Col } \\
(n=11)\end{array}$ & $\begin{array}{c}\text { Carr } \\
(n=46)\end{array}$ & $\begin{array}{c}\text { Carr }+ \text { Col } \\
(n=41)\end{array}$ \\
\hline Volume $(\mathrm{mL})$ & $2.6 \pm 0.2 \dagger$ & $5.7 \pm 0.3 \dagger$ & $3.5 \pm 0.1$ & $7.5 \pm 0.3 \dagger$ \\
Cell count $\left(10^{6} / \mathrm{mL}\right)$ & $3.0 \pm 0.8 \dagger$ & $1.6 \pm 0.2 \dagger$ & $20.0 \pm 1.9$ & $5.7 \pm 0.7 \dagger$ \\
WBC/WBC $+\mathrm{RBC}$ & $0.83 \pm 0.05$ & $0.93 \pm 0.04$ & $0.91 \pm 0.02$ & $0.87 \pm 0.03$ \\
PMN/WBC & $0.23 \pm 0.05 \dagger$ & $0.50 \pm 0.06 \dagger$ & $0.81 \pm 0.02$ & $0.51 \pm 0.04 \dagger$ \\
PMN/pouch $\left(10^{6}\right)$ & $0.9 \pm 0.2 \dagger$ & $4.3 \pm 0.8 \dagger$ & $48.8 \pm 4.9$ & $18.8 \pm 2.9 \dagger$
\end{tabular}

* Values are mean \pm SEM of data from all experiments on pouch fluid at $6 \mathrm{~h}$ postchallenge with normal saline for injection. Col. Carr, or Carr + Col. WBC, white blood cells; RBC, red blood cells.

$+p<0.001$ vs Carr. 
Table 2. Anti-inflammatory effects of Col compared with systemic dexamethasone or indomethacin*

\begin{tabular}{lcccc} 
Pouch fluid & $\begin{array}{c}\text { Carr } \\
(n=16)\end{array}$ & $\begin{array}{c}\text { Carr }+ \text { Col } \\
(n=13)\end{array}$ & $\begin{array}{c}\text { Carr }+ \text { Dex } \\
(n=16)\end{array}$ & $\begin{array}{c}\text { Carr }+ \text { Ind } \\
(n=13)\end{array}$ \\
\hline Volume $(\mathrm{mL})$ & $3.4 \pm 0.2$ & $7.8 \pm 0.7 \dagger$ & $1.9 \pm 0.2 \dagger \ddagger$ & $3.4 \pm 0.2 \ddagger$ \\
Cell count $\left(10^{6} / \mathrm{mL}\right)$ & $19.5 \pm 2.8$ & $2.4 \pm 0.5 \dagger$ & $13.3 \pm 2.4 \ddagger$ & $3.7 \pm 1.0 \dagger$ \\
WBC/WBC $+\mathrm{RBC}$ & $0.98 \pm 0.01$ & $0.97 \pm 0.02$ & $0.93 \pm 0.05$ & $0.97 \pm 0.01$ \\
PMN/WBC & $0.86 \pm 0.02$ & $0.42 \pm 0.05 \dagger$ & $0.90 \pm 0.05 \ddagger$ & $0.58 \pm 0.11 \S$ \\
PMN/pouch $\left(10^{6}\right)$ & $53.2 \pm 6.6$ & $8.0 \pm 2.9 \dagger$ & $18.9 \pm 3.3 \dagger \ddagger$ & $8.7 \pm 3.0 \dagger$ \\
\hline
\end{tabular}

* Values are mean \pm SEM of rat air pouch fluid parameters at $6 \mathrm{~h}$ postchallenge with Carr alone, or with Carr plus Col, Carr plus systemic dexamethasone (Dex), or Carr plus indomethacin (Ind). WBC, white blood cells; RBC, red blood cells.

$+p<0.001$ us Carr.

$\ddagger p<0.05$ vs Carr + Col.

$\S p<0.05$ vs. Carr.

and $6.70 \times 10^{9} \mathrm{PMN} / \mathrm{L}$ in colostrum. The stability of human PMN in colostrum or serum in vitro appears somewhat different, but this difference would not account for the large decreases in PMN counts seen in animals receiving carrageenan plus colostrum.

$P M N$ granule marker assay on pouch fluid. An initial experiment using lysozyme as a PMN granule content marker revealed high levels of lysozyme activity in aqueous colostrum and in colostrum-treated pouch fluid and poor correlation with PMN counts in pouch fluid. Aqueous colostrum contained modest $\beta$ glucuronidase activity, and this PMN granule marker correlated well with PMN count in carrageenan and carrageenan plus colostrum-treated pouch fluid. In a single experiment, mean $\beta$ glucuronidase activity was $7.6 \pm 2.8$ fluorescence units per 25 $\mu \mathrm{L}$ (units) $(n=2)$ in normal saline-treated pouch fluid samples, $34.2 \pm 1.7$ units ( $n=2, p=0.02$ versus normal saline) in colostrum-treated pouch fluid samples, $95.1 \pm 14.1$ units $(n=$ $7)$ in carrageenan-treated pouch fluid samples, and $54.9 \pm 5.4$ units ( $n=5, p=0.04$ versus Carr) in carrageenan plus colostrumtreated pouch fluid samples. If lysis of PMN was occurring in colostrum-treated pouches, increasing amounts of PMN granule contents would be seen as cells arrived and were lysed. This action would result in increased $\beta$-glucuronidase activity per observed leukocyte in colostrum-treated pouch fluids, independent of any added $\beta$-glucuronidase activity due to the colostrum itself. In pouch fluid from carrageenan-challenged animals, the relationship between $\beta$-glucuronidase activity and the observed PMN count was $2.28 \pm 0.56$ units per $10^{6} \mathrm{PMN} / \mathrm{mL}(r=0.875$, $n=7, p=0.01$ ) using linear regression analysis. In samples from animals challenged with carrageenan plus colostrum, the same relationship was $2.84 \pm 0.50$ units per $10^{9} \mathrm{PMN} / \mathrm{L}(r=0.956$. $n=5, p=0.01)$. The difference between these two slopes was not significant $(p=0.49)$, suggesting that PMN granule contents were not accumulating in carrageenan plus colostrum-treated pouches as more PMN arrived.

Anti-inflammatory activity of molecular sizing fractions of aqueous colostrum. Separation of a pool of aqueous colostrum by molecular sizing filtration yielded three fractions. Treatment with unfractionated colostrum from the same pool decreased PMN influx after carrageenan challenge from $25.7 \pm 6.6 \times 10^{6}$ $\mathrm{PMN} /$ pouch to $2.4 \pm 0.3 \times 10^{6}$ (Carr $+\mathrm{Col}, n=3$, versus Carr, $n=4, p=0.03$ ). Treatment with the colostrum fraction with components $>100000 \mathrm{D}$ produced a PMN influx of $30.6 \pm 3.8$ $\times 10^{6} \mathrm{PMN} /$ pouch $(n=3, p=0.6$ versus Carr $)$. Treatment with the fraction with components from 100000 to $10000 \mathrm{D}$ produced a PMN influx of $20.2 \pm 0.8 \times 10^{6} \mathrm{PMN} /$ pouch $(n=3$, $p=0.5$ versus Carr). Treatment with the fraction with components $<10000 \mathrm{D}$ produced a PMN influx of $6.9 \pm 4.0 \times 10^{6}$ $\mathrm{PMN} /$ pouch ( $n=3, p=0.08$ versus Carr). Although the number of animals is small and significant suppressive activity of any fraction cannot be shown, these data suggest that anti-inflammatory activity in colostrum may be segregated to the low molecular weight fraction and that proinflammatory activity may be segregated to the high molecular weight fraction.

\section{DISCUSSION}

The rat air pouch model used in these studies is able to contain colostrum, challenge materials, and products of the inflammatory process for prolonged periods in contact with an organized tissue surface in vivo. Compared with other rodent models, this model can demonstrate inflammatory responses with good sensitivity (28-30). The model has been used previously to demonstrate the effects of known anti-inflammatory agents (31). Our experiments used observations of PMN numbers in pouch fluid as an end point because this parameter is closely associated with acute inflammation in vivo and can be estimated accurately and reproducibly in the laboratory. Our conclusions would not be altered if leukocyte influx instead of PMN influx into pouch fluid was used as the primary end point.

Carrageenan was used as the inflammatory challenge in these experiments because it is well studied and predictable in animal models of inflammation, and in preliminary trials it produced an impressive $\mathrm{PMN}$ response at an early point in acute inflammation. The diverse properties of carrageenan have been previously reviewed (32). Although gross signs of inflammation were infrequent, carrageenan challenge produced a significant cellular influx at $6 \mathrm{~h}$ postchallenge that was consistent with an acute inflammatory response. We conclude that carrageenan injection induces a prompt local acute inflammatory response in the rat air pouch.

For colostrum to protect newborns from inflammation, others have suggested that it first must be a poor inducer of inflammation (25). In these experiments, the inflammatory activity of colostrum was intermediate compared with the activity of normal saline or of carrageenan. Colostrum was less inflammatory than laboratory-prepared PBS that was used as a negative control in a single preliminary trial (data not shown) and that probably contained small amounts of endotoxin. We conclude that the aqueous phase of human colostrum is a poor inducer of acute inflammation in this model. The modest cellular response to aqueous colostrum injection might be related to the known chemoattractant activity of the major milk protein casein. The persistence of fluid in colostrum-injected pouches may reflect the higher osmotic activity of colostrum compared with the other challenge materials.

Pooled aqueous human colostrum significantly suppressed PMN influx after carrageenan challenge in these experiments. The degree of suppression was equivalent to those of potent antiinflammatory agents given systemically. The decreases seen in the presence of colostrum are most likely due to suppression of a component of the inflammatory cascade leading to PMN accumulation, because incubation of $\mathrm{PMN}$ in colostrum in vitro and correlation of leukocyte granule contents and cell counts in pouch fluid samples revealed no signs of significant PMN lysis. We conclude that colostrum can significantly alter acute inflammation in response to carrageenan challenge in the rat air pouch.

Whether decreases in the inflammatory response seen with colostrum treatment are the result of a specific anti-inflammatory activity was not examined. If colostrum possessed a specific 
anticarrageenan effect, then decreased PMN influx would be expected with colostrum treatment. However, in a small number of animals, suppression of PMN influx in response to heat-killed Staphylococcus aureus in tryptic soy broth also was observed when colostrum was injected with the staphylococcal preparation, as well as when an anti-inflammatory agent was given (data not shown). Although colostrum possesses secretory $\operatorname{IgA}$ and lysozyme, which might decrease the inflammatory response to staphylococci, it is unlikely that anti-inflammatory effects after carrageenan and staphylococcal challenge are the result of separate specific interactions with these stimuli. Therefore, we believe the aqueous phase of colostrum is a potent suppressor of acute inflammation in this in vivo model. These data directly support the hypothesis that human colostrum is anti-inflammatory in vivo and indirectly supports the hypothesis that colostrum may protect newborns from exposure to PMN.

Variation in the anti-inflammatory activity among individual donor's colostrum is evident and suggests that this anti-inflammatory activity is related to specific components in colostrum that vary among mothers. The identity of these anti-inflammatory components in colostrum is unclear. Fractionation of colostrum by molecular size filtration suggested that colostral antiinflammatory factors may segregate with low molecular mass components (less than $10000 \mathrm{D}$ ). These data also could be interpreted as reflecting partition of proinflammatory colostral components, such as casein, to the high molecular mass fraction. Digestion of colostrum with proteinase $\mathrm{K}$ did not eliminate the suppression of PMN influx in one experiment. and PAGE of the digested colostrum showed disappearance of proteins with molecular masses greater than $50000 \mathrm{D}$ (data not shown). The suppressive activity of this digested colostrum was greater than undigested colostrum, suggesting a loss of proinflammatory activity with protease treatment. Proinflammatory or chemotactic activity in colostrum may obscure some anti-inflammatory activity seen in this model, and this chemotactic activity may be due to casein. Lactoferrin is a milk component that can decrease the production of proinflammatory cytokines such as IL-1 and tumor necrosis factor by mononuclear cells (33), as well as the production of complement component C 3 convertase (34), and so could suppress inflammation. However, lactoferrin should partition to the 10000 - to $100000-\mathrm{D}$ colostrum fraction and should be proteinase $\mathrm{K}$-susceptible and thus is unlikely to be the active component in this model. Additional studies to delineate and characterize the mediators of this anti-inflammatory effect are needed.

These data show that human colostrum has an anti-inflammatory effect at the surface of a subcutaneous pouch in rats. If human milk is important in prevention of diarrheal disease and damage from PMN in infants, we would hypothesize that it also has an anti-inflammatory effect at the mucosal surface of the gastrointestinal tract in newborns. Because this is not a model of the mucosal inflammation, the biologic significance of this antiinflammatory effect is unclear. We believe these results suggest the need for examination of the effects of human milk colostrum on inflammation in more biologically relevant settings.

In summary, a rat subcutaneous air pouch model of inflammation using carrageenan as an inflammatory stimulus was used to test the effects of the aqueous phase of colostrum on inflammation. Pooled aqueous colostrum had little acute inflammatory activity, and when present in the pouch with carrageenan, it suppressed acute inflammation as effectively as systemically administered dexamethasone or indomethacin. These data are the first experimental evidence of an anti-inflammatory effect of colostrum in vivo. They are consistent with in vitro data suggesting that human milk or colostrum has antileukocyte activity and epidemiologic data suggesting anti-inflammatory effects.

Acknowledgments. The authors thank Elizabeth Cramer and Irene Townsend for their enthusiastic efforts at collecting colos- trum, Sarah Mcllheran and Mirta Machado for technical assistance, and Carol Wolf at Merck Sharp \& Dohme for supplying anti-inflammatory agents.

\section{REFERENCES}

1. Ho FCS, Wong RLC, Lawton JWM 1979 Human colostral and breast milk cells. Acta Paediatr Scand 68:389-396

2. Crago SS, Prince SJ. Pretlow TG, McGhee JR. Mestecky J 1979 Human colostral cells. Clin Exp Immunol 38:585-597

3. Kohl S, Pickering LK. Cleary TC. Steinmetz KD. Loo LS 1980 Human colostral cytotoxicity. J Infect Dis 142:884-891

4. Ho PC. Lawton JMW 1978 Human colostral cells: phagocytosis and killing of $E$ coli and C: albicans. J Pediatr 93:910-915

5. Khan AJ, Rosenfeld W, Vadapalli M. Biagton J, Khan P. Hug A. Evans HE 1980 Chemotaxis and random migration of human milk cells. J Pediatr 96:879-882

6. Thorpe LW, Rudloff HE, Powell LC. Goldman AS 1986 Decreased response of human milk leukocytes to chemoattractant peptides. Pediatr Res 20:373377

7. Pickering LK. Cleary TG, Kohl S, Getz S 1980 Polymorphonuclear leukocytes of human colostrum. J Infect Dis 142:685-693

8. Johnson DF. France GL. Marmer DJ. Steele RW 1980 Bactericidal mechanisms of human breast milk leukocytes. Infect Immun 28:314-318

9. Keller MA. Kidd RM, Bryson YJ. Turner JL. Carter J 1981 Lymphokine production by human milk lymphocytes. Infect Immun 32:632-636

10. Weiss SJ 1989 Tissue destruction by neutrophils. N Engl J Med 320:365-376

11. Foster SJ, Cunliffe CJ. McCormick ME 1988 Polymorphonuclear leukocyte induced damage to the articular cartilage in acute immunologic arthritis in rabbits. Biochem Pharmacol 37:1181-1183

12. Hernandez LA. Grisham MB. Twohig B. Arfors KE. Harlan JM. Granger DN 1987 Role of neutrophils in ischemia-reperfusion-induced microvascular injury. Am J Physiol 253:H699-H703

13. Giannella RA 1979 Importance of the intestinal inflammatory reaction in Salmonella-mediated intestinal secretion. Infect Immun 23:140-145

14. Pickering LK. Cleary TC, Caprioli RM 1983 Inhibition of human polymorphonuclear leukocyte function by components of human colostrum and mature milk. Infect Immun 40:8-15

15. Crago SS, Kulhavy R. Prince SJ, Mestecky J 1981 Inhibition of the pokeweed mitogen-induced response of normal peripheral blood lymphocytes by humoral components of colostrum. Clin Exp Immunol 45:386-392

16. Hooton JWL. Pabst HF. Spandy DW. Paetkau V 1991 Human colostrum contains an activity that inhibits the production of IL-2. Clin Exp Immunol 86:520-524

17. Buescher ES, Mcllheran SM 1988 Antioxidant properties of human colostrum. Pediatr Res 24:14-19

18. Buescher ES. Mcllheran SM. Frenck RW 1989 Further characterization of human colostral antioxidants. Pediatr Res 25:266-270

19. Cunningham AS. Jelliffe DB. Jelliffe EFP 1991 Breast-feeding and health in the 1980s: a global epidemiologic review. J Pediatr 118:659-666

20. Glass RI. Stoll BJ 1989 The protective effect of human milk against diarrhea. Acta Paediatr Scand [Suppl] 351:131-136

21. Goldman AS. Goldblum RM 1990 Human milk: immunologic-nutritional relationships. Ann NY Acad Sci 587:236-245

22. Goldman AS. Thorpe LW, Goldblum RM. Hanson LA 1986 Anti-inflammatory properties of human milk. Acta Paediatr Scand 75:689-695

23. Mata LJ. Urrutia JJ, Garcia B. Fernandez R. Behar M 1969 Shigella infection in breast-fed Guatemalan Indian neonates. Am J Dis Child 117:142-146

24. Mata LJ. Urrutia JJ 1971 Intestinal colonization of breast-fed children in a rural area of low socioeconomic level. Ann NY Acad Sci 176:93-109

25. Lucas A. Cole TJ 1990 Breast milk and neonatal necrotizing enterocolitis. Lancet 336:1519-1523

26. Boyum A 1986 Isolation of mononuclear cells and granulocytes from human blood. Scand J Clin Invest 21(suppl 97):77-89

27. Suzuki K. Swenson C. Sasagawa S. Sakatani T. Watanabe M. Kobayashi M. Fujikura T 1983 Age-related decline in lysozomal enzyme release from polymorphonuclear leukocytes after N-formyl-methionyl-leucyl-phenylalanine stimulation. Exp Hematol 11:1005-1013

28. Sedgwick AD, Sin YM. Edwards JCW. Willoughby DA 1983 Increased inflammatory reactivity in newly formed lining tissue. J Pathol 141:483-495

29. Balasubramaniam GS. Hurley JV 1987 The structure and reaction of the microcirculation in a subcutaneous air pouch in the rat. $J$ Pathol 151:139146

30. Sedgwick AD, Lees $P 1986$ A comparison of air pouch, sponge and pleurisy models of acute carrageenan inflammation in the rat. Agents Actions 18:439446

31. Al-Duaij AY, Sedgwick AD. Willoughby DA 1986 The effect of anti-inflammatory and rheumatoid disease modifying drugs on prolonged immune and non-immune inflammation in the six-day air pouch of rats. Int $\mathrm{J}$ Tissue React 8:463-468

32. Di Rosa M 1972 Biological properties of carrageenan. J Pharm Pharmacol 24:89-102

33. Crouch SPM. Slater KJ. Fletcher J 1992 Regulation of cytokine release from mononuclear cells by the iron-binding protein lactoferrin. Blood 80:235240

34. Kijlstra A. Jeurissen SHM 1982 Modulation of classical C3 convertase of complement by tear lactoferrin. Immunology 47:263-270 